\title{
An autotuning procedure for motion control of oscillatory mechatronic systems
}

\author{
Marco Giacomelli \\ Department of Mechanical and Industrial Engineering \\ University of Brescia, m.giacomelli009@unibs.it \\ Giovanna Finzi \\ Department of Mechanical and Industrial Engineering \\ University of Brescia, giovanna.finzi@unibs.it \\ Luca Simoni \\ Department of Mechanical and Industrial Engineering \\ University of Brescia, luca.simoni@unibs.it \\ Davide Colombo \\ Gefran Drives and Motion Srl \\ Gerenzano (VA), Italy, davide.colombo@gefran.com \\ Vlastimil Šetka \\ Department of Cybernetics \\ University of West Bohemia, setka@ntis.zcu.cz \\ Antonio Visioli \\ Department of Mechanical and Industrial Engineering \\ University of Brescia, antonio.visioli@unibs.it
}

This is the pre-peer reviewed version of the following article: An autotuning procedure for motion control of oscillatory mechatronic systems overhead cranes, which has been published in final form at

DOI: 10.1109/ETFA.2019.8869039. This article may be used for non-commercial purposes in accordance with Journal terms and conditions for Self-Archiving.

\begin{abstract}
In this paper an automatic tuning procedure for a cascade position control architecture of general mechatronic systems is presented. The proposed procedure firstly estimates the transfer function of the system by analysing its open-loop response to a signal specifically designed in order to satisfy the system position, velocity and torque constraints. Then, if present, oscillatory behaviours are detected by finding the (anti)resonances of the frequency response. Parametric tuning rules are eventually defined for the PID controllers and for the biquadratic filters. Results obtained by implementing the procedure on a Hardware In the Loop setup demonstrate the effectiveness of the method.
\end{abstract}

\section{Index Terms}

Autotuning, mechatronic systems, PID controllers, oscillations.

\section{INTRODUCTION}

The tuning of Proportional-Integral-Derivative (PID) controllers is a crucial task when approaching a new system. While experienced operators can effectively refine the tuning of controller parameters depending on the specific application, it is generally non-trivial to guess a first set of values. Moreover, when the system to be controlled is subject to varying dynamics over the time, for example mechatronics systems with varying inertia loads, a change in the controllers parameters has to be done frequently, so that an automated tuning would be desirable.

Many PID autotuning techniques for process control applications can be found in the literature [1]. On the contrary, autotuning techniques for the control of mechatronic systems are still a relevant research topic [2]. The generalization of the process control autotuning procedures to the control of mechatronic systems is generally limited by some important differences between the two fields of application. In particular, mechatronic systems are characterized by constraints on torque, acceleration, velocity and position that can depend on the actuator, on the application or on both of them. Moreover, mechatronic systems are affected 
by a series of nonlinearities like static and dynamic friction, backslash etc. which complicates the autotuning procedure.

Nonetheless, an advantage of this kind of systems is that a generic mechatronic system can be described, for control purposes, as a series of bodies and transmissions characterized by inertias and elasticities; an automatic approach for the tuning of the controllers for mechatronic systems should therefore exploit this model during the identification phase.

In any case, an autotuning procedure should satisfy some common requirements, such as a minimum involvement of the operator (who, ideally, should not be involved at all in the procedure) and a minimum execution time, in order to reduce the overall commissioning costs.

In the context of autotuning for mechatronic systems, various techniques have been proposed in the recent years. In [3]-[5] variations of Pseudo-Random Binary Signals (PRBS) are applied as a torque input to the systems in order to excite all the frequencies. The input torque signal and the output signal (which can be either a velocity or position signal measured on the motor or on the load side) are then processed using a simple Fast Fourier Transform (FFT) method or the Welch method [3]. While this approach gives good results on unconstrained systems, the limits of the system are generally not taken into account when defining the input signal.

An alternative to PRBS signal is the use of a swept sine wave with frequencies that vary in the range of interest of the specific application. In [6], [7] a methodology that exploits the swept sine wave deals with non-linearities and measurement noise.

In [8] a two stage approach is proposed. First, an estimation of the parameters that characterize the slow dynamics of the system (inertia, static and viscous frictions) is obtained by filtering a PRBS signal with a low-pass filter. Then, the high frequencies dynamics parameters (that is, the elasticities) are estimated using a non-filtered PRBS signal. Also in this case, the constraints are ignored, limiting the applicability of the procedure to unconstrained systems.

Once a model of the system is obtained (usually, in the form of a transfer function), the controller parameters have to be tuned. In [9], [10] PID controllers are tuned in order to explicitly compensate for the presence of oscillations. Another approach, used in [11], is to delete the oscillatory dynamics of the system by filtering the command signal with low-pass, notch or bi-quadratic filter.

Regarding the tuning of the PID parameters, an optimization based procedure is proposed in [8]. Although an optimization brings a good control performance, it usually involves a computational effort that is not sustainable by industrial drives and PLCs.

In this paper, we extend the method presented in [12], where an autotuning procedure for the velocity control of a general mechatronic system is proposed. This procedure explicitly takes into account the (torque, velocity and position) limits of the system, designing an open-loop exciting signal accordingly to those limits and by assuming matched load and motor inertias as explained in [13]. Although the results of [12] were promising, we extend the methodology by modifying the procedure, changing the definition of the exciting signal mixing it with a PRBS approach and by adding the tuning of the position control loop and of filters that delete the oscillations on the load side. The transfer function identification is also modified by fusing the analytical approach of [6] with a nonlinear optimization approach for a rapid convergence. The proposed autotuning methodology has been implemented on a Hardware-In-the-Loop (HIL) setup, and experimental results are here presented.

The paper is structured as follows: in Section II the proposed autotuning methodology is delineated showing its flowchart and the various steps are explained in detail; in Section III the experimental setup is described, while in Section IV the obtained experimental results are shown and analysed. Section $\mathrm{V}$ concludes the paper.

\section{Proposed Methodology}

The flowchart of the proposed procedure is shown in Figure 1. First, an initial acquisition of the velocity of the system is analysed in order to determine the noise affecting its measurement. Then, a torque ramp with a slight gradient is given to the system in order to identify the static friction torque $\hat{K}_{f}$, determined as the value of the torque when the measured velocity overcomes the maximum estimated value of the noise for a meaningful number of acquisitions. The input slope gradient $\Delta \tau$ can easily be defined as a percentage of the full-scale torque $\tau_{\max }$, so that

$$
\Delta \tau=\frac{\tau_{\max }}{N_{s}}
$$

where $N_{s}$ is the number of sampling periods necessary to reach the maximum torque $\tau_{\max }$. In this paper the value of $N_{s}$ is arbitrarily set to 10000 . This value that can be increased or decreased depending on the relevance of the static friction on the particular system.

After having estimated $\hat{K}_{f}$, an input signal is given to the system in order to obtain and analyse its response. To generate the input torque/force signal, we suppose to have some position, speed and torque constraints denoted as $\bar{\theta}, \dot{\dot{\theta}}$ and $\tau_{\text {max }}$, respectively. The open -loop torque signal to give to the system is computed as shown in Figure 2 . Different cycles are repeated with different applied torques until the identification time exceeds the maximum identification time $T_{\text {end }}$, defined as the inverse of the required frequency resolution $\Delta f$. In particular, for each cycle, at the beginning, the cycle torque $\tau_{n e w}$ is computed randomly between the estimated static friction torque value $\hat{K}_{f}$ and the maximum applicable torque $\tau_{\max }$. Then, a cycle based on the following four parts is performed:

- positive torque $\tau_{\text {new }}$, 


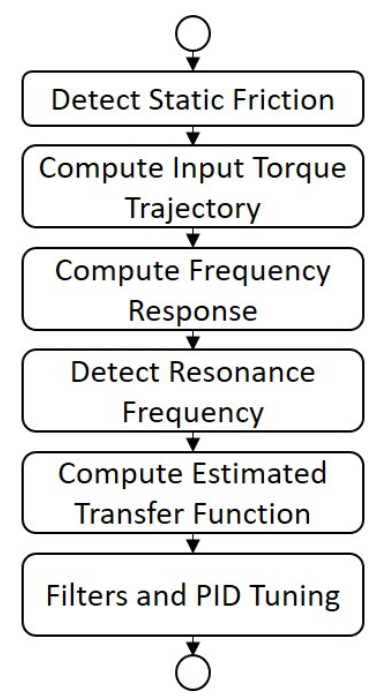

Fig. 1. Flowchart of the proposed autotuning procedure.

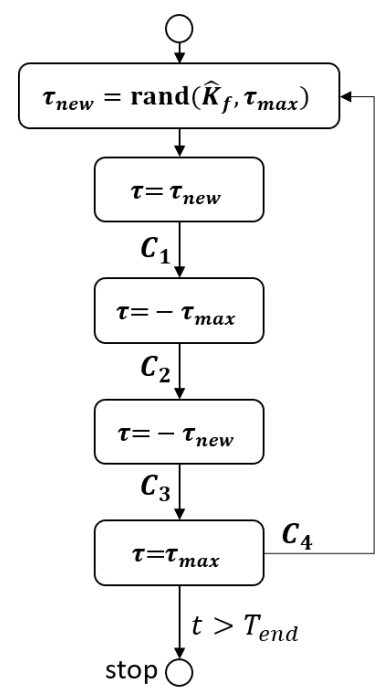

Fig. 2. Input torque trajectory generation flowchart.

- negative maximum torque $-\tau_{\max }$,

- negative torque $-\tau_{\text {new }}$,

- positive maximum torque $\tau_{\max }$.

In particular, the maximum cycle torque $\tau_{n e w}$ is applied to the system until the first condition (namely $C_{1}$ ) is met; then a deceleration phase with maximum negative torque is performed until condition $C_{2}$ is met; after that, a negative maximum cycle torque is applied to the system until condition $C_{3}$ is met; finally, a negative deceleration phase with maximum positive torque is performed until condition $C_{4}$ is met o until the stop signal is received. These conditions are set in order to satisfy the imposed constraints.

In particular, condition $C_{1}$ is verified when the current speed overcomes the maximum speed or when the actual position is equal to half of the distance between the initial position $\theta_{0}$ and the maximum one. Condition $C_{2}$ is verified when the current speed becomes negative. Condition $C_{3}$ is the symmetric of $C_{1}$. Condition $C_{4}$ is verified when the current speed becomes 


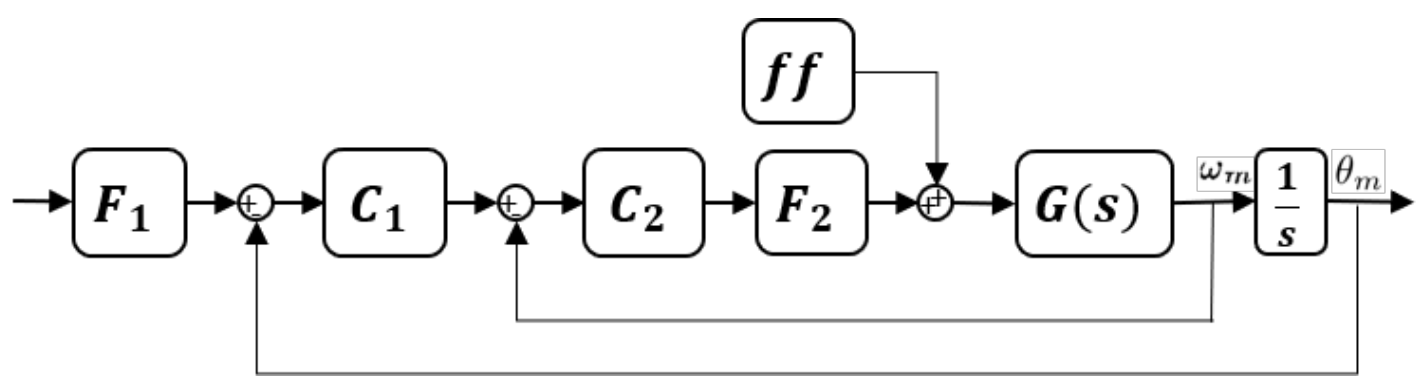

Fig. 3. Proposed position control scheme: cascade control with PID controllers $C_{1}, C_{2}$, the biquadratic filters $F_{1}, F_{2}$ and the friction feedforward compensator $f f$.

positive again. Formally, it is

$$
\begin{aligned}
& C_{1}=\left\{\begin{array}{l}
\text { TRUE if } \dot{\theta} \geq \overline{\dot{\theta}} \text { OR } \theta \geq \frac{\bar{\theta}+\theta_{0}}{2}-\theta_{0} \\
\text { FALSE otherwise }
\end{array}\right. \\
& C_{2}=\left\{\begin{array}{l}
\text { TRUE } \text { if } \dot{\theta} \leq 0 \\
\text { FALSE otherwise }
\end{array}\right. \\
& C_{3}=\left\{\begin{array}{l}
\text { TRUE if } \dot{\theta} \leq-\dot{\theta} \text { OR } \theta \leq-\left(\frac{\bar{\theta}+\theta_{0}}{2}-\theta_{0}\right. \\
\text { FALSE otherwise }
\end{array}\right. \\
& C_{4}=\left\{\begin{array}{l}
\text { TRUE if } \dot{\theta} \geq 0 \\
\text { FALSE otherwise }
\end{array}\right.
\end{aligned}
$$

The measured velocity is stored together with the applied torque signal from which the static friction torque $\hat{K}_{f}$ is subtracted, as the model that will fit the data acquisitions does not include the presence of nonlinearities. These data are then analysed with the Welch method [14] using a Blackman windowing, obtaining an estimation of their spectral density. The transfer function between the torque input and the velocity of the motor is then estimated using a nonlinear Levenberg-Marquardt optimization algorithm [15]. While in [3] the same approach is used for identifying the analytical form of the transfer function, the choice for the initial values of the model parameters is unclear. As the Levenberg-Marquardt algorithm might find a local minumum, a wrong choice of the initial values can bring to the wrong estimation of the transfer function.

For the correct determination of a good set of initial parameters for the optimization algorithm, the resonance and antiresonance peaks of the spectral density are identified by detecting the maximum of the product of the prominence and the width of each phase peak. Then, the resonance and antiresonance frequencies can be approximately identified assuming that at those frequencies their phase is the peak phase minus half of its prominence. Then, the frequency of the mechanical pole of the system can be identified as the frequency for which the magnitude of the spectral density decreases of more than $3 \mathrm{~dB}$. Once those three points have been found, an analytical solution for the identification of the transfer function is presented in [6]. Due to possible noise on the spectral density data, this method can give unreliable results, in particular regarding the system low-frequency gain. Nonetheless, the parameters resulting from this analytical solution represent a good starting point for the nonlinear Levenberg-Marquardt optimization.

The theoretical transfer function between torque and motor velocity of a two mass system, identified with the aforementioned techniques, is in the form:

$$
G(s)=\frac{K}{s+p} \frac{s^{2}+2 \xi_{a} \omega_{a} s+\omega_{a}^{2}}{s^{2}+2 \xi_{r} \omega_{r} s+\omega_{r}^{2}} .
$$

Once the model of the system has been obtained, the effects of the oscillatory mode can be compensated using bi-quadratic filters. The proposed control architecture, shown in Figure 3, has two bi-quadratic filters $F_{1}$ and $F_{2}$, one filtering the setpoint and the other one filtering the output of the internal velocity PID controller. The bi-quadratic filter acting inside the velocity control loop deletes the system resonance and antiresonance, obtaining the normalized filter

$$
F_{2}(s)=\frac{\omega_{a}^{2}}{\omega_{r}^{2}} \frac{s^{2}+2 \xi_{r} \omega_{r} s+\omega_{r}^{2}}{s^{2}+2 \xi_{a} \omega_{a} s+\omega_{a}^{2}}
$$

so that, defining

$$
\bar{K}:=K \frac{\omega_{a}^{2}}{\omega_{r}^{2}}
$$


the filtered system transfer function becomes of first order in the form

$$
G(s) F_{2}(s)=\frac{\bar{K}}{s+p} .
$$

Subsequently, a velocity PI controller in the form

$$
C_{2}(s)=K_{p_{2}}\left(1+\frac{1}{T_{i_{2}} s}\right)
$$

is tuned based on (5) imposing a phase margin $\phi_{m}$ and a cutoff frequency $\omega_{c}$. The resulting PI parameters are

$$
\begin{aligned}
T_{i_{2}} & =\frac{\tan \left(\phi_{m}-\frac{\pi}{2}+\arctan \left(\frac{\omega_{c}}{p}\right)\right)}{\omega_{c}} \\
K_{p_{2}} & =\frac{T_{i_{2}}}{\bar{K} \sqrt{1+T_{i_{2}}^{2} \omega_{c}^{2}}\left(\omega_{c}^{4}+\omega_{c}^{2} p^{2}\right)}
\end{aligned}
$$

While the closed loop control of the motor can guarantee good performance on the motor side, the performance on the load side could even decrease if only the motor is taken into account. In fact, a resonance is present in the transfer function that describes the relation between the position of the motor and the position of the load. Even if this transfer function can not be identified as, in the great majority of mechatronic systems, there is no sensor on the load side, its resonance can be obtained knowing that, when the system is controlled in open loop, it deletes the antiresonance in (3). Therefore, in order to delete this resonance, the setpoint is filtered by a second bi-quadratic filter $F_{1}$. The parameters of $F_{1}$ can be easily obtained by imposing the same zeros of (3) and two real poles that have the same distance from the origin with respect to the two complex zeros, obtaining the normalized filter

$$
F_{1}(s)=\frac{s^{2}+2 \xi_{a} \omega_{a} s+\omega_{a}^{2}}{s^{2}+2 \omega_{a} s+\omega_{a}^{2}} .
$$

Finally, the position PID controller need to be tuned. In accordance to the prescriptions that can be found in literature, the outer control loop of a cascade control should be at least five times slower than the internal loop. Choosing a proportional position controller, the position loop cutoff frequency $\omega_{c_{p}}$ can be set as, for example, one tenth of the velocity loop cutoff frequency $\omega_{c_{v}}$. The position controller $C_{1}$ is therefore chosen as a proportional controller

$$
C_{1}(s)=K_{p_{1}} .
$$

By imposing

$$
\omega_{c_{p}}=0.1 \omega_{c_{v}}
$$

we obtain

$$
K_{p_{1}}=\frac{1}{K_{p_{2}} \bar{K}} \sqrt{\frac{\omega_{c_{p}}^{4} T_{i_{2}}^{2}\left(P+K_{p_{2}} \bar{K}\right)^{2}+\omega_{c_{p}}^{2}\left(K_{p_{2}} \bar{K}+T_{i_{2}} \omega_{c_{p}}^{2}\right)^{2}}{T_{i_{2}}^{2} \omega_{c_{p}}^{2}+1}} .
$$

Moreover, as shown in Figure 3, a feedforward signal compensates for the static friction: being $\dot{\theta}_{\text {ref }}$ the reference velocity of the internal loop, the feedforward signal $f f$ is calculated as

$$
f f=\hat{K}_{f} \operatorname{sign}\left(\dot{\theta}_{r e f}\right) \text {. }
$$

\section{EXPERIMENTAL SETUP}

The proposed autotuning procedure has been tested on a HIL setup. The experimental setup consists of two brushless servomotors coupled by means of a rigid transmission, two Gefran ADV200S servo drives, a Real-time Controller with REXYGEN software platform [16] and a standard PC that runs Matlab ${ }^{\mathrm{TM}}$ 2017b. The Real-Time Controller is implemented on hardware developed as "Building Block 1" in the context of a project called I-MECH [17], and communicates with Gefran Drives by means of the EtherCAT protocol, while communication with the Matlab script running on the standard PC use HTTP REST API provided by REXYGEN. The generation of the exciting signal is managed by the drivers, which send to the controller the measured motor position and velocity, the torque signal and the state of the procedure over the EtherCAT with $1 \mathrm{~ms}$ period. REXYGEN is responsible for the storing of the measurements and for the direct communication with the drives, setting a flag for the execution of the autotuning procedure and changing the control parameters after the procedure is completed. The stored signals are sent to Matlab by means of a HTTP request over standard Ethernet and TCP/IP. The elaboration of the signals and the computation of the model of the system and the control parameters is managed in Matlab environment on a standard PC, simulating the presence of a centralized supervisor which is responsible for the autotuning 


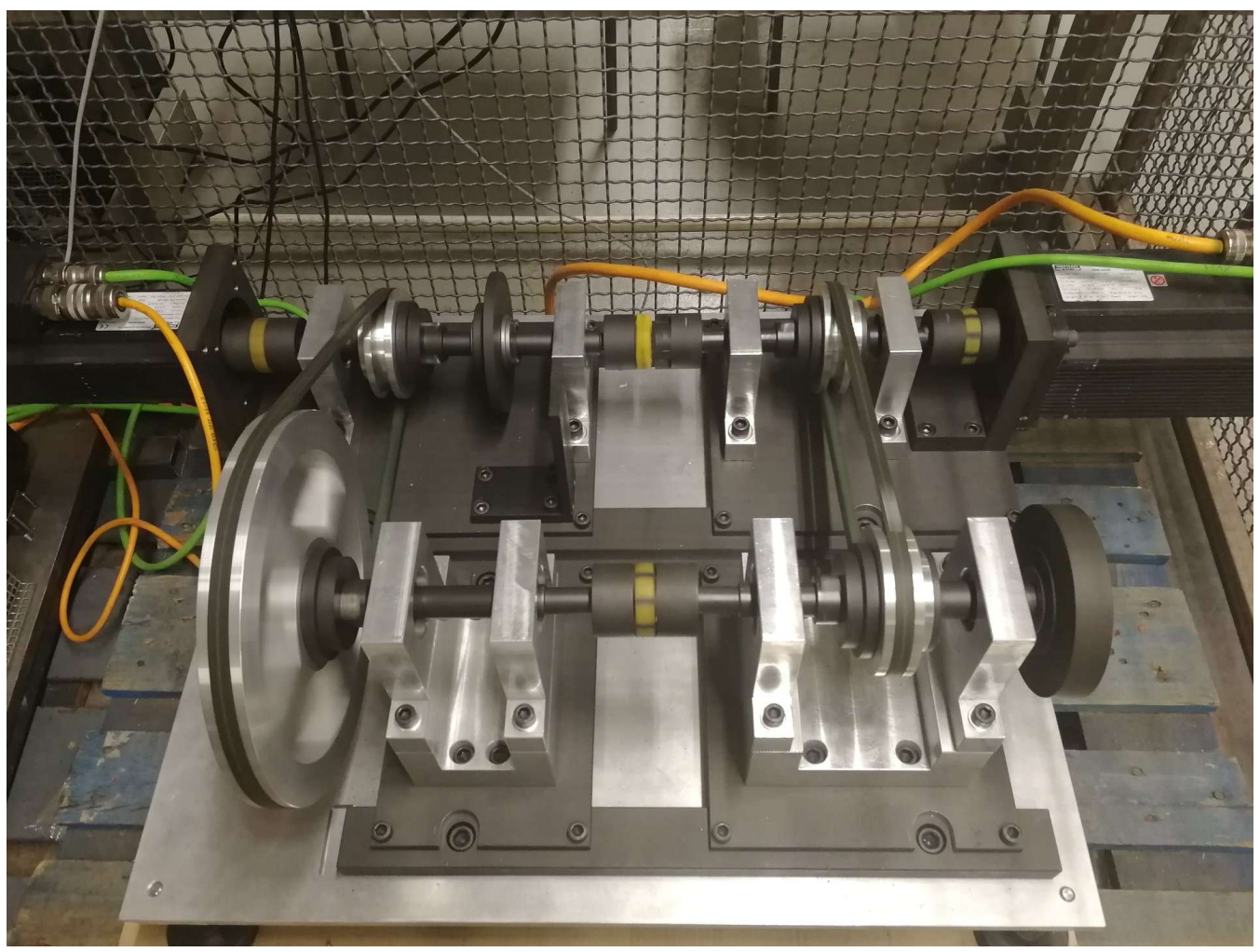

Fig. 4. Experimental HIL setup.

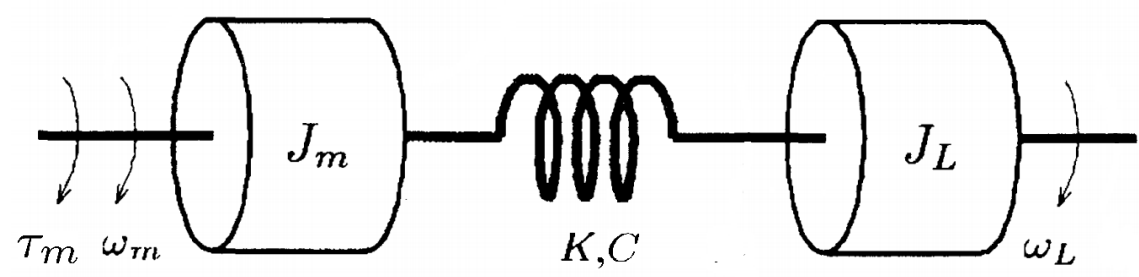

Fig. 5. Two-inertia system.

procedure and data analysis. The whole procedure is completely automatic and is activated by launching a Matlab script. It has to be noted that the code running on Matlab has been tested for auto code generation compatibility and could, for example, run into REXYGEN-based device or other industrial controllers only, so that the particular environment on which it runs does not entail a loss of generality, meaning that the procedure retains its orientation to industrial field applications.

The experimental setup is shown in Figure 4. The presence of a second motor allows the simulation of a generic mechatronic systems: the second motor can indeed react to a variation of its velocity opposing the reaction torque of the mechatronic system, whose dynamics is simulated in real-time on the driver.

For the purpose of testing the autotuning procedure, the simulated system consists of a two-inertia system, like that shown in Figure 5, characterized by an elastic transmission with a viscous friction $C$ and an elasticity $K$ that connects the motor with an inertia of $J_{m}$ to a load of inertia $J_{L}$; a viscous friction with a coefficient $B_{m}$ is applied to the rotation of the load. Moreover, a static friction with a coefficient $K_{f}$ is present. For a more realistic approach, the system is also characterized by limits on its maximum position displacement $\theta_{\max }$, its maximum velocity $\dot{\theta}_{\max }$ and the maximum motor torque $\tau_{\max }$. The model that has just been described is a standard model for a generic mechatronic system with one oscillatory mode. The parameters of the simulated system are shown in Table I. The correspondence between the model in Table I and the system running on the HIL setup has been obtained by compensating the internal frictions of the experimental setup. The transfer function of the system, considered the torque as the input and the velocity as the output, can be written in the form (3) as

$$
G(s)=\frac{126.58\left(s^{2}+0.3797 s+125.9\right)}{(s+0.1709)\left(s^{2}+0.9304 s+253.1\right)} .
$$




\begin{tabular}{llll}
\hline Parameter & Value & Parameter & Value \\
\hline$C$ & 0.003 & $K$ & 1.0 \\
$J_{m}$ & $0.0079 \mathrm{kgm}$ & $J_{L}$ & $0.0079 \mathrm{kgm}$ \\
$B_{m}$ & 0.0027 & $K_{f}$ & 0.3 \\
$\theta_{\max }$ & $300 \mathrm{rad}$ & $\dot{\theta}_{\text {max }}$ & $280 \mathrm{rad} / \mathrm{s}$ \\
$\tau_{\max }$ & $5 \mathrm{Nm}$ & & \\
\hline
\end{tabular}

TABLE I

PARAMETERS OF THE SYSTEM SIMULATED ON THE HIL SETUP.
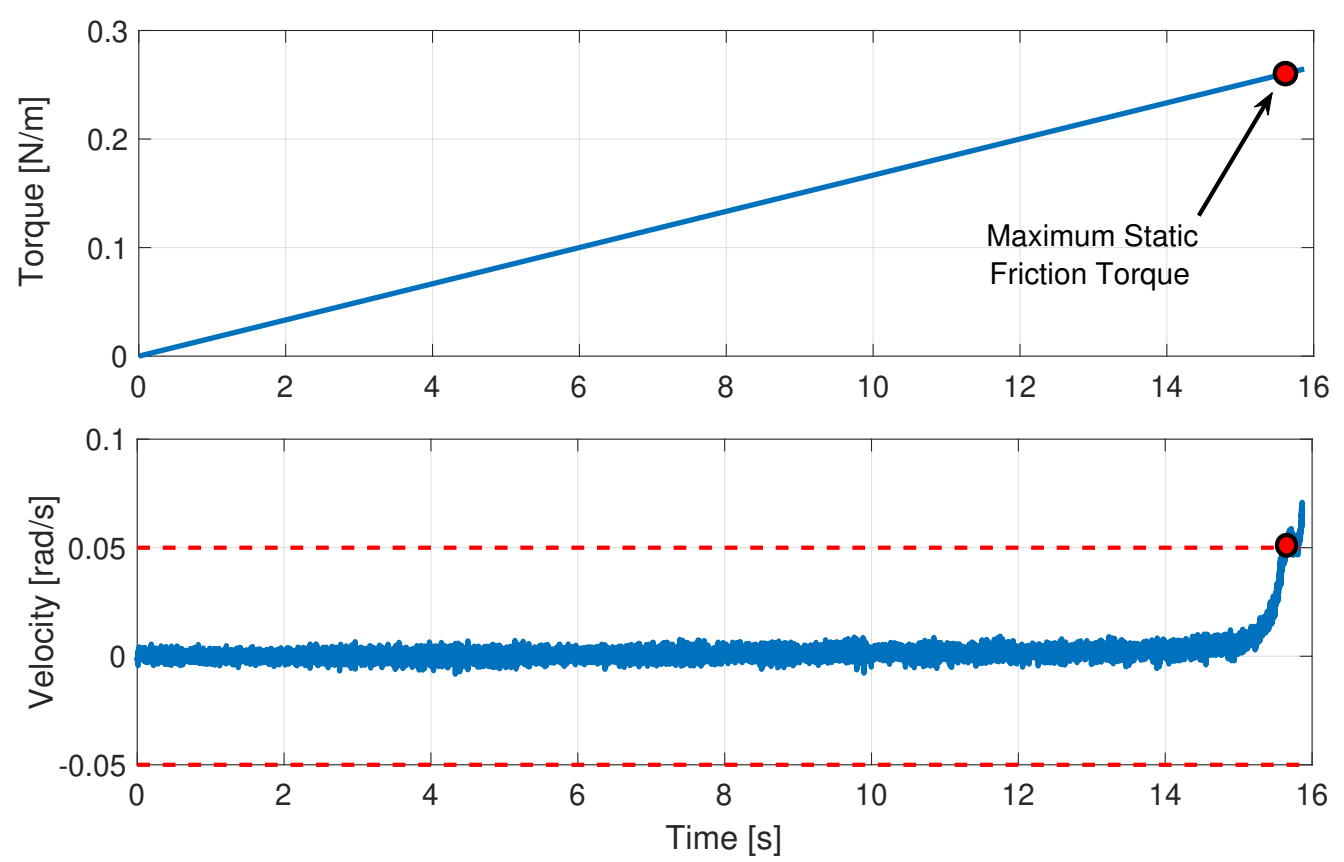

Fig. 6. Static friction identification phase. The maximum static friction torque is identified as the torque given to the system when the velocity exceeds the limits (dashed red line), which value is calculated as the maximum value of the noise on the velocity multiplied by a safety factor of 5 .

\section{EXPERIMENTAL RESULTS}

The autotuning procedure proposed in Section II has been implemented on the HIL setup, and the results are shown hereafter. As the maximum torque of the motor is $\tau_{\max }=5 \mathrm{Nm}$, the static friction identification phase obtained by setting $N_{s}=10000$ in (1) is shown in Figure 6. The resulting identified static friction coefficient is $\hat{K}_{f}=0.2603 \mathrm{Nm}$. The identification procedure is then executed for a total time $T_{\text {end }}=80 \mathrm{~s}$, which gives a frequency resolution of

$$
\Delta f=\frac{1}{T_{\text {end }}}=0.0125 \mathrm{~Hz}
$$

The signals are sampled with a sampling period of $T_{s}=0.001 \mathrm{~s}$, which, for the Nyquist sampling criterion, gives a maximum identifiable frequency of $500 \mathrm{~Hz}$. Nonetheless, the obtained spectral information with the Welch method has been limited in the range $[0.0125,100] \mathrm{Hz}$, being $100 \mathrm{~Hz}$ a reasonable upper limit for a digital filter running on a $1 \mathrm{~ms}$ sampling period controller. The trajectory of the system is shown in Figure 7. It must be noted that, as expected, the limits of the systems are always respected during the trajectory.

From the spectral density resulting from the Welch analysis, shown in Figure 8, the three points needed for the analytical transfer function identification of [6] are identified (red squares in Figure 8). The resulting transfer function parameters are then used as the initial guess for a Levenberg-Marquardt optimization, which results in the transfer function shown in Figure 8 , which can be expressed in the form (3) as

$$
\hat{G}(s)=\frac{92.724\left(s^{2}+0.6957 s+125.9\right)}{(s+0.1996)\left(s^{2}+0.3407 s+258.5\right)} .
$$

In Figure 8 the identified system $\hat{G}$ is compared with the spectral density resulting from the Welch analysis and the theoretical system transfer function $G$. 

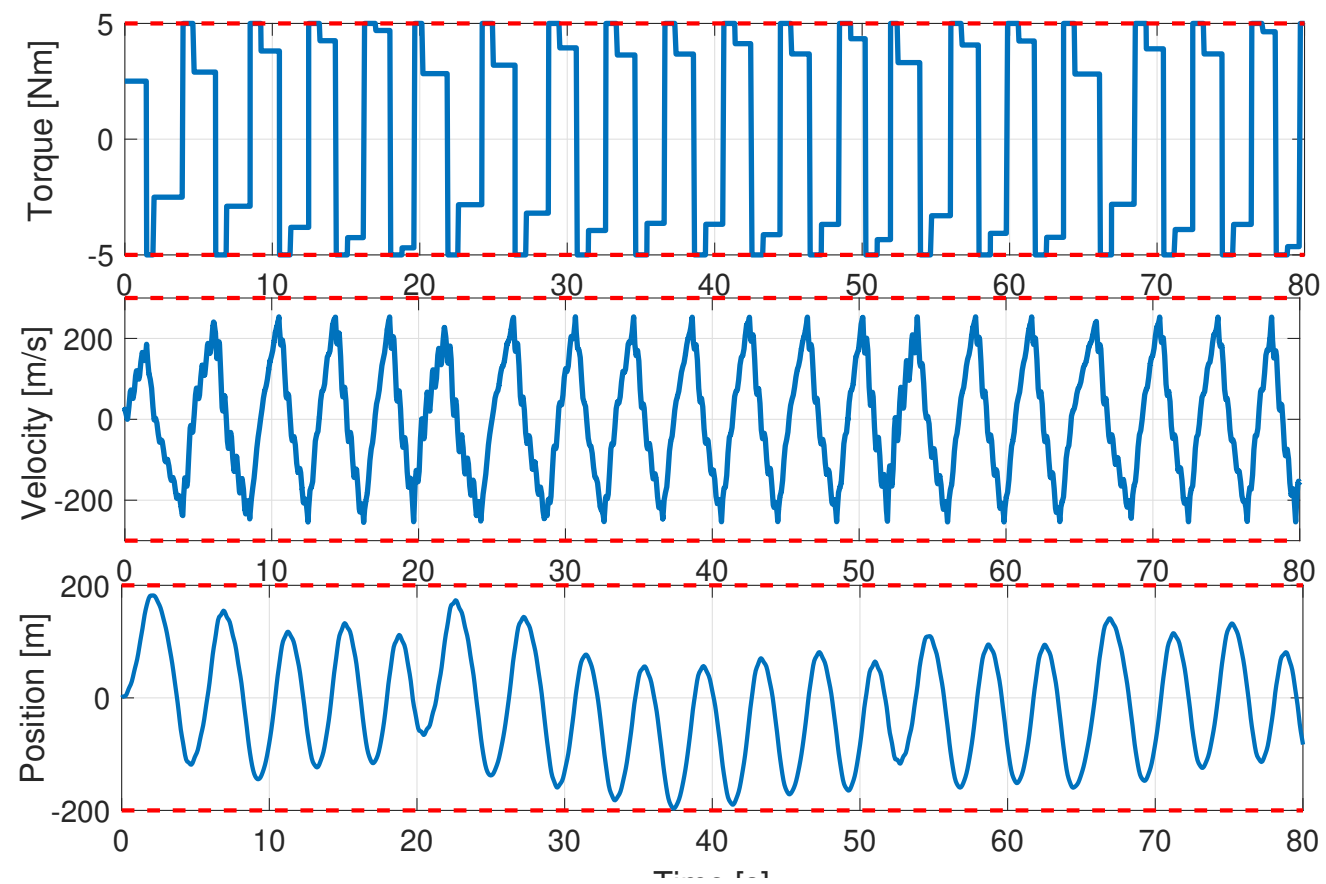

Fig. 7. Trajectory of the system during the autotuning procedure. The limits of the system are shown in dashed red line.
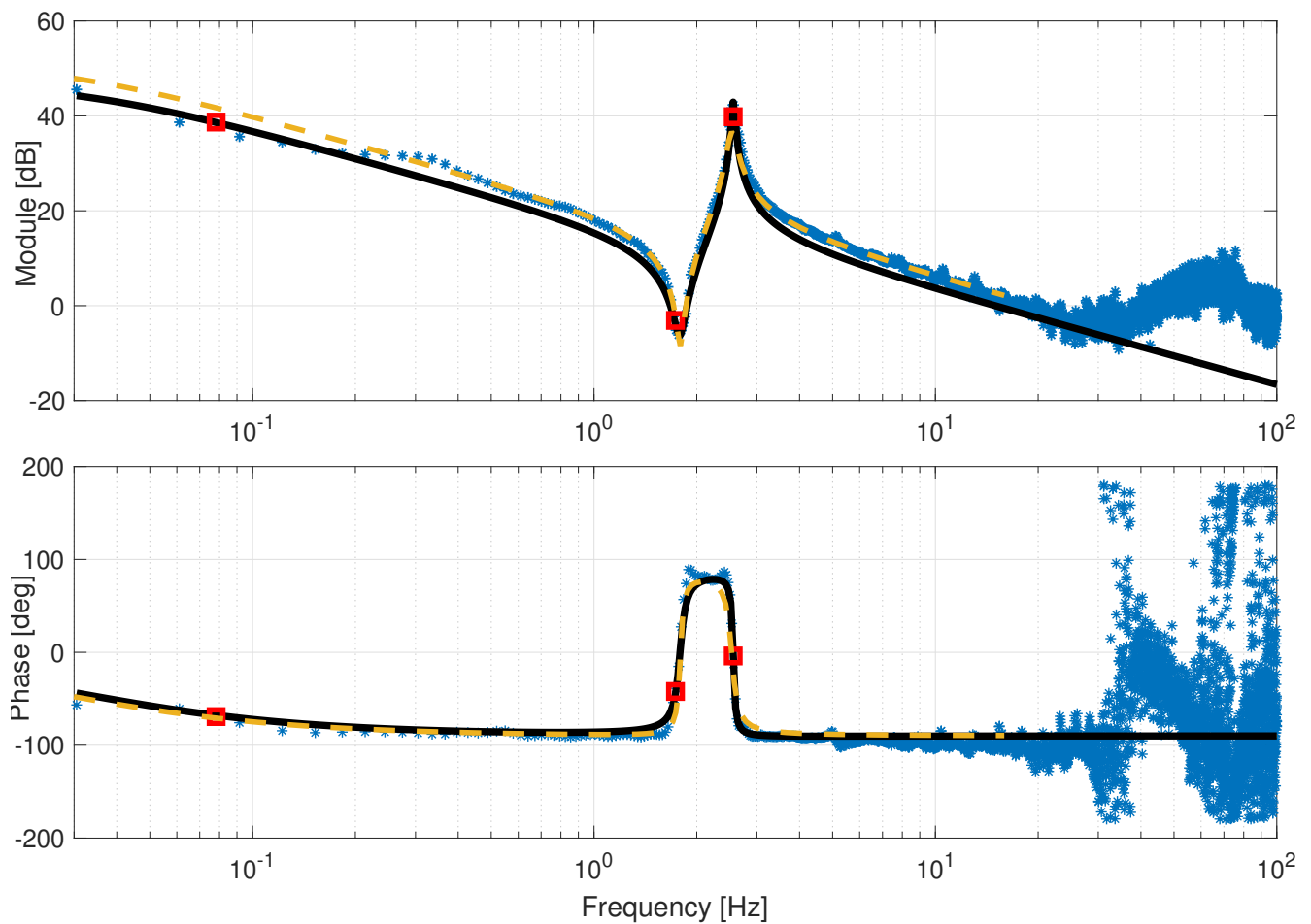

Fig. 8. Bode diagrams of the system. The theoretical transfer function $\hat{G}$ (yellow dashed line), the spectral density obtained with the Welch method (blue stars), the three points for the analytical identification (red squares) and the identified system after the optimization (black solid line). 
With the estimated transfer function of the system, the velocity cutoff frequency $\omega_{c_{v}}$ and phase margin $\phi_{m}$ are set to $\omega_{c_{v}}=30$ $\mathrm{rad} / \mathrm{s}$ and $\phi_{m}=85^{\circ}$, and the velocity PI controller results in

$$
C_{2}(s)=0.6614\left(1+\frac{1}{0.3539 s}\right)
$$

while the filters result in

$$
F_{2}(s)=\frac{0.487 s^{2}+0.1659 s+125.9}{s^{2}+0.6957 s+125.9}
$$

and

$$
F_{1}(s)=\frac{s^{2}+0.6957 s+125.88}{s^{2}+22.44 s+125.88} .
$$

The position controller is obtained with (9) and results in

$$
C_{1}(s)=3.1646 \text {. }
$$

The position closed loop control of the system is obtained by filtering the position reference, following the scheme in Figure 3.

In order to verify the performance of the autotuning procedure, a series of steps has been given as a reference signal to the system. It has to be noted that, for the position control of mechatronic systems, steps in the reference are normally avoided in conditions of normal operation, as they usually generates saturations on the actuators, and other trajectories are preferred (e.g. 7 traits trajectories). Nonetheless, the analysis of the step responce is a de-facto benchmark test for the analysis of system dynamics, and it is here used in order to address the closed-loop dynamics of the controlled system. Figure 9(a) shows the position response of the system with the tuned velocity and position PID controllers, but without the filters $F_{2}$ and $F_{1}$. The elasticity of the transmission generates a series of oscillations on the load side. In Figure 9(b) the filters $F_{2}$ and $F_{1}$ are activated, and the oscillation is greatly reduced both on motor and on load side.

\section{CONCLUSIONS}

In this paper a new procedure for the cascade control autotuning of a general oscillating mechatronic system has been presented. In this procedure, once the static friction is detected, an open loop procedure is employed, where a signal that explicitly takes into account the system limits such as maximum torque, velocity and position is used to excite the system dynamics. The spectral density of the system is obtained with the Welch method with a Blackman windowing of the input signal and the corresponding velocity trajectory. The (anti-)resonance frequencies are detected by analysing prominence and width of the phase response; an analytical method first and a nonlinear optimization secondly give the identified transfer function of the system, which is then used to tune the velocity and position controllers as well as the filters which delete the oscillatory response of the system. The application of the proposed procedure on a HIL setup shows its effectiveness in reducing load oscillations without sensors on the load side and respecting the imposed limits during the identification procedure.

Future developments of the proposed procedure could include the autotuning of multi-modes oscillatory systems and the definition of a performance assessment strategy that will activate autonomously the autotuning procedure when the performance degrades.

\section{REFERENCES}

[1] K. J. Åström and T. Hägglund, Advanced PID Control. Research Triangle Park, USA: ISA Press, 2006.

[2] M. Cech, A. J. Beltman, and K. Ozols, "I-MECH, Smart System Integration for Mechatronic Applications," in Proceedings 24th IEEE International Conference on Emerging Technologies and Factory Automation, Zaragoza, E, 2019.

[3] S. Villwock and M. Pacas, "Application of the welch-method for the identification of two-and three-mass-systems," IEEE Transactions on Industrial Electronics, vol. 55, no. 1, pp. 457-466, 2008.

[4] J. Weissbacher, E. Grunbacher, and M. Horn, "Automatic tuning of a servo drive speed controller for industrial applications," in Proceedings IEEE International Conference on Mechatronics, Vicenza, I, 2013, pp. 700-705.

[5] S. Beineke, F. Schutte, H. Wertz, and H. Grotstollen, "Comparison of parameter identification schemes for self-commissioning drive control of nonlinear two-mass systems," in Proceedings Annual Meeting IEEE Industry Applications Society, New Orleans, LA, 1997, pp. 493-500.

[6] M. Goubej, A. Krejci, and M. Schlegel, "Robust frequency identification of oscillatory electromechanical systems," in Proceedings 19th International Conference on Process Control, Strbske Pleso, SK, 2013.

[7] M. Goubej, "Kalman filter based observer design for real-time frequency identification in motion control systems," in Proceedings 20th International Conference on Process Control, Strbske Pleso, SK, 2015.

[8] M. Calvini, M. Carpita, A. Formentini, and M. Marchesoni, "PSO-based self-commissioning of electrical motor drives," IEEE Transactions on Industrial Electronics, vol. 62, no. 2, pp. 768-776, 2015.

[9] M. Goubej and M. Schlegel, "Robust PID control of electrical drive with compliant load," in Proceedings 19th IFAC World Congress, Cape Town, SA, 2014, pp. 11781-11786.

[10] G. Zhang and J. Furusho, "Speed control of two-inertia system by PI/PID control," IEEE Transactions on Industrial Electronics, vol. 41, no. 3, pp. 603-609, 2000. 

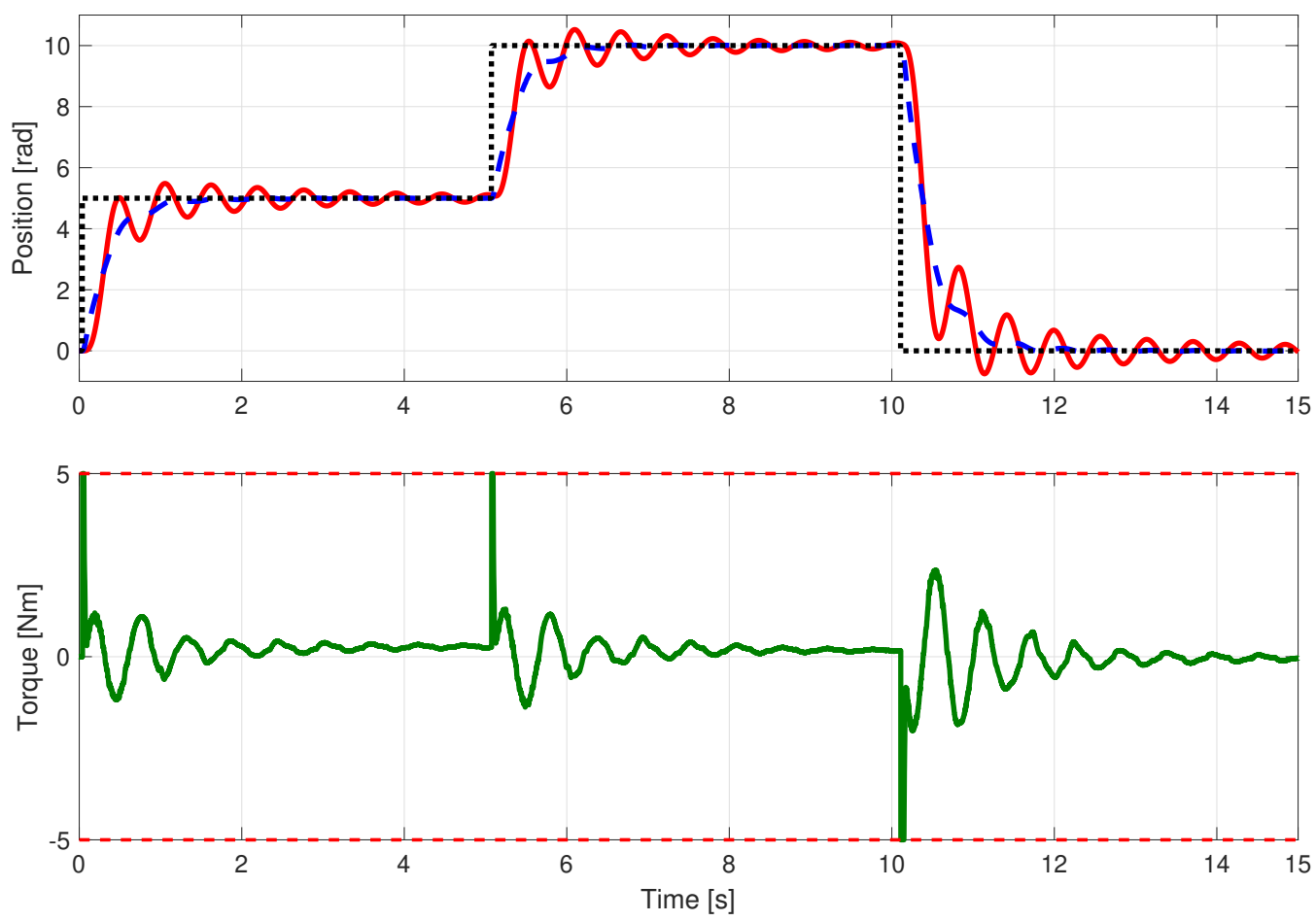

(a) Closed loop position control without filters.
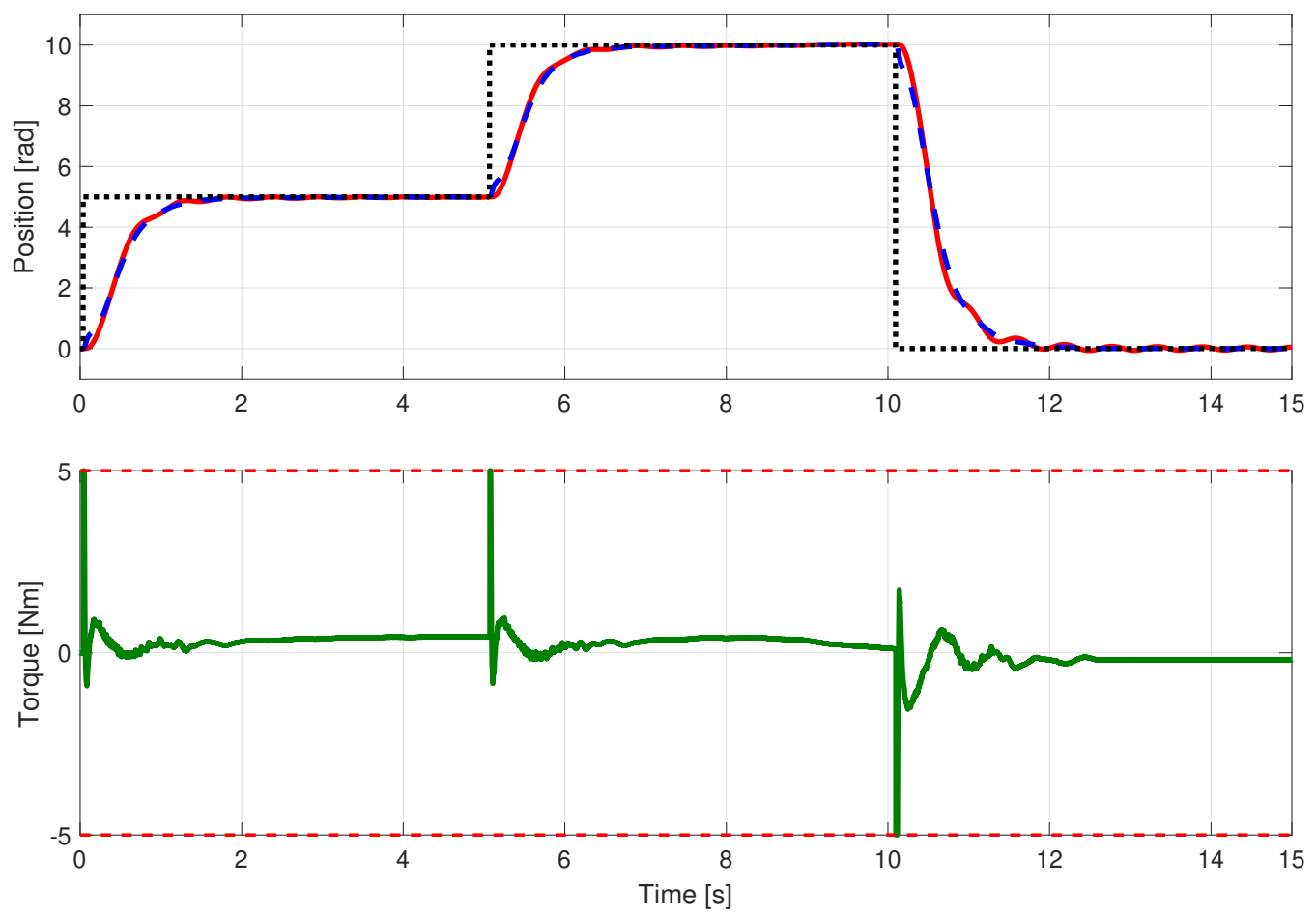

(b) Closed loop position control with filters.

Fig. 9. Step response of the closed loop control with (a) and without (b) the filters. Position reference (black dotted line), motor position (blue dashed line), load position (red solid line) and motor torque (green solid line). 
[11] G. Ellis and R. D. Lorenz, "Resonant load control methods for industrial servo drives," in Proceedings IEEE Industry Applications Conference, Rome, I, 2000, pp. 1438-1445.

[12] M. Giacomelli, D. Colombo, L. Simoni, G. Finzi, and A. Visioli, "A fast autotuning method for velocity control of mechatronic systems," IFACPapersOnLine, vol. 51, no. 4, pp. 208-213, 2018.

[13] R. W. Armstrong, "Load to motor inertia mismatch: Unveiling the truth," in Drives and Controls Conference, 1998.

[14] P. Welch, "The use of fast fourier transform for the estimation of power spectra: a method based on time averaging over short, modified periodograms," IEEE Transactions on audio and electroacoustics, vol. 15, no. 2, pp. 70-73, 1967.

[15] J. J. Moré, "The levenberg-marquardt algorithm: implementation and theory," in Numerical analysis. Springer, 1978, pp. 105-116.

[16] O. Severa and M. Čech, "Rex-rapid development tool for automation and robotics," in Proceedings 8th International Conference on Mechatronic and Embedded Systems and Applications, 2012, pp. 184-189.

[17] “I-MECH, Smart Mechatronic Solutions," https://www.i-mech.eu/, accessed: 2019-04-12. 